\title{
473586 - EMG DETECTION OF LOSS OF CONSCIOUSNESS DURING A STANDARDIZED IV INDUCTION
}

\author{
Jagpaul Sahota, MD ${ }^{1}$, Henrik Huttunen, MD, FRCPC ${ }^{1}$, Ali Zandi, MASc ${ }^{3}$, Stephane \\ Bibian, MASc, $\mathrm{PhD}^{2}$, Patrick Yu, MD, FRCPC ${ }^{1}$, Guy Dumont, $\mathrm{PhD}^{3}$, Craig Ries, \\ M.D., FRCPC, Ph.D. ${ }^{1}$ \\ 1. Department of Anesthesiology, Pharmacology \& Therapeutics, University of \\ British Columbia, Vancouver, BC, Canada \\ 2. Cleveland Medical Devices Inc, Cleveland, OH, USA \\ 3. Department of Electrical \& Computer Engineering UBC Faculty of Applied \\ Science, University of British Columbia, Vancouver, BC, Canada
}

Introduction: The electromyogram (EMG) that is generated by the frontalis muscle is traditionally viewed by neurologists as a contaminate for the electroencephalogram (EEG). During the 1980s, however, an anesthesia monitor was developed that was based on the facial EMG. In view of the ongoing controversy for the role of the EMG with current anesthesia EEG monitors, we wished to determine if the sudden loss of consciousness during a controlled intravenous induction of general anesthesia could be detected by a decrease in EMG spectral power.

Methods: We analyzed an EEG/EMG database that our group had previously collected with approval from the UBC clinical research ethics board and written patient consent. In this database, 35 ASA I-II patients had received a standardized intravenous induction for tracheal intubation. The induction consisted of a fentanyl bolus $\left(3 \mathrm{mcg} / \mathrm{kg}^{-1}\right)$ and was followed $60 \mathrm{~s}$ later by a propofol infusion (2 mg/ $\mathrm{kg}^{-1}$ over $\left.30 \mathrm{~s}\right)$. A loss of count (LOC) was used to determine loss of consciousness, at which time a rocuronium bolus (0.6 $\mathrm{mg} / \mathrm{kg}^{-1}$ ) was administered followed by intubation 60 seconds later. Continuous digital heart rate and two-channel EEG monitoring were recorded in a double-blinded fashion and transmitted wirelessly to a laptop computer. The EEG/EMG was then analyzed using power calculation, coefficient of variation, receiver operator characteristic curve and prediction probability analysis using the PK statistic.

Results: The mean EMG power (dB) in the 60 second time period post-fentanyl and prepropofol was $36 \mathrm{~dB}$, with a coefficient of variation of $15 \%(n=35)$. Based on a receiver operating characteristic curve for the EMG diagnosis of LOC, an EMG power of 30dB had a sensitivity of 1 and a specificity of 0.97 (Figure). The prediction probability for this event was 0.90 .

Discussion: The EMG has strong predictive value for determining LOC in a controlled IV induction in healthy patients. Based on previously published probabilities, this compares favorably with the WAV $(\mathrm{PK}=0.975)$ and the $\mathrm{BIS}(\mathrm{PK}=0.89)^{1}$.

References: Anesthesiology 2006;105(4):A1030. 


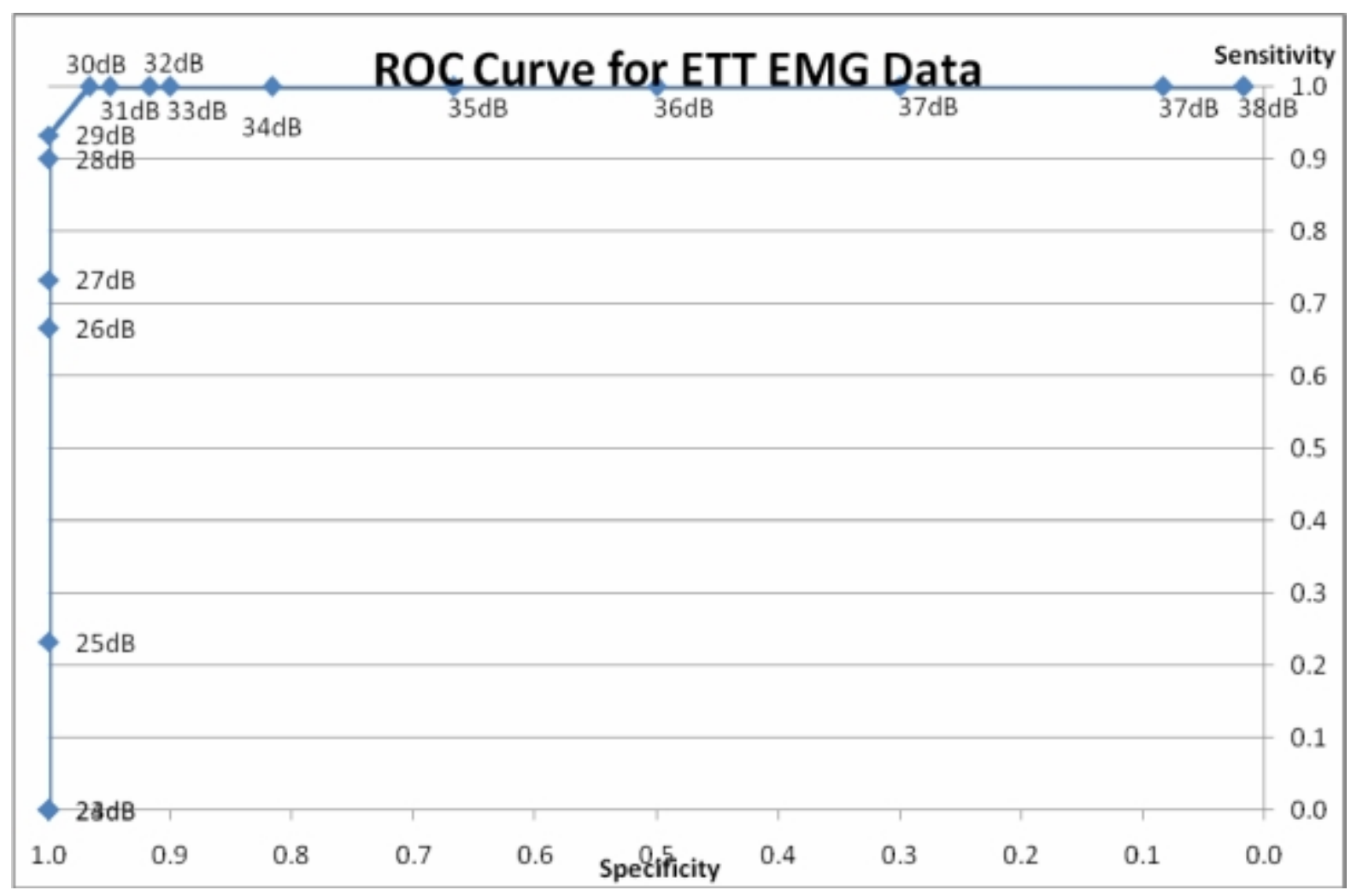

